Case Report

\title{
Pneumonia due to a Rare Pathogen: Achromobacter xylosoxidans, Subspecies denitrificans
}

\author{
Hesham Awadh, ${ }^{1}$ Munthir Mansour, ${ }^{1}$ Obadah Aqtash, ${ }^{1}$ and Yousef Shweihat ${ }^{2}$ \\ ${ }^{1}$ Internal Medicine Department, Marshall University Joan C. Edwards School of Medicine, Huntington, WV, USA \\ ${ }^{2}$ Pulmonology Department, Marshall University Joan C. Edwards School of Medicine, Huntington WV, USA \\ Correspondence should be addressed to Hesham Awadh; awadh@live.marshall.edu
}

Received 24 February 2017; Accepted 18 July 2017; Published 15 August 2017

Academic Editor: Larry M. Bush

Copyright @ 2017 Hesham Awadh et al. This is an open access article distributed under the Creative Commons Attribution License, which permits unrestricted use, distribution, and reproduction in any medium, provided the original work is properly cited.

\begin{abstract}
Achromobacter xylosoxidans, subspecies denitrificans, is a gram-negative rod recently implicated as an emerging cause of infection in both immunosuppressed and immunocompetent populations. Few cases are reported in literature involving multiple body systems. Diagnosis depends on cultures of appropriate specimens, and management usually is by administration of appropriate antibiotics (usually agents with antipseudomonal activity). We report a rare case of pneumonia due to infection with this organism, in a patient with preexisting bronchiectasis secondary to chronic aspiration.
\end{abstract}

\section{Introduction}

Achromobacter xylosoxidans subspecies denitrificans is a gram-negative bacillus recently emerging as a causative agent of infection [1]. The Achromobacter species has many subspecies the most clinically important of which are xylosoxidans and denitrificans [1]. There are infrequent reports of infections with this organism involving various organs (Table 1). It seems that a dysfunctional immune status and/or prior structural damage plays a role in the pathogenicity Achromobacter xylosoxidans, subspecies denitrificans. We report a rare case of pneumonia due to this organism in a 45year-old female with bronchiectasis secondary to recurrent aspiration.

\section{Case Presentation}

This is a 45-year-old White female with past medical history of asthma and gastroesophageal reflux disease (GERD) treated with Nissen fundoplication in the past. She presented to our clinic with chronic cough productive of greenish sputum. She improved with previous antibiotic use of levofloxacin on several occasions but her symptoms would recur as soon as she stops the antibiotics. Chest $\mathrm{X}$-ray at initial evaluation showed an infiltrate bilaterally more pronounced on the right lower lobe. A CT scan of the chest was obtained and confirmed the infiltrates and showed bronchiectatic changes bilaterally in the lower lobes (Figure 1). Her autoimmune screen came back negative for rheumatoid arthritis and Sjogren's syndromes, yet her immunoglobulins were elevated and her alpha one antitrypsin and immunoglobulin $\mathrm{E}$ ( $\mathrm{IgE}$ ) were at normal levels. Her sweat chloride test was normal. Chronic recurrent aspiration was suspected and an esophageal $\mathrm{PH}$ monitor along with esophageal manometry confirmed our suspicion of chronic aspiration secondary to severe acid reflux with elevated DeMeester score. Bronchoscopy was performed to rule out an obstructive disease and to obtain samples to rule out mycobacterial disease. Cultures came back positive for heavy growth of a nonfermenter later identified as Achromobacter xylosoxidans, subspecies denitrificans. Sensitivities were evaluated and the bacteria was sensitive to levofloxacin, amikacin, cefepime, ceftazidime, gentamicin, meropenem, piperacillin/tazobactam, tobramycin, and trimethoprim-sulfamethoxazole. It was found to be resistant to aztreonam, cefotaxime, and ciprofloxacin, with incubation period of 5 days. Airway clearance techniques with percussion and flutter valve and bronchodilator therapy with hypertonic saline nebulizers were initiated. She was started on a 3week course of levofloxacin but her symptoms recurred one month after stopping the antibiotic despite airway clearance 


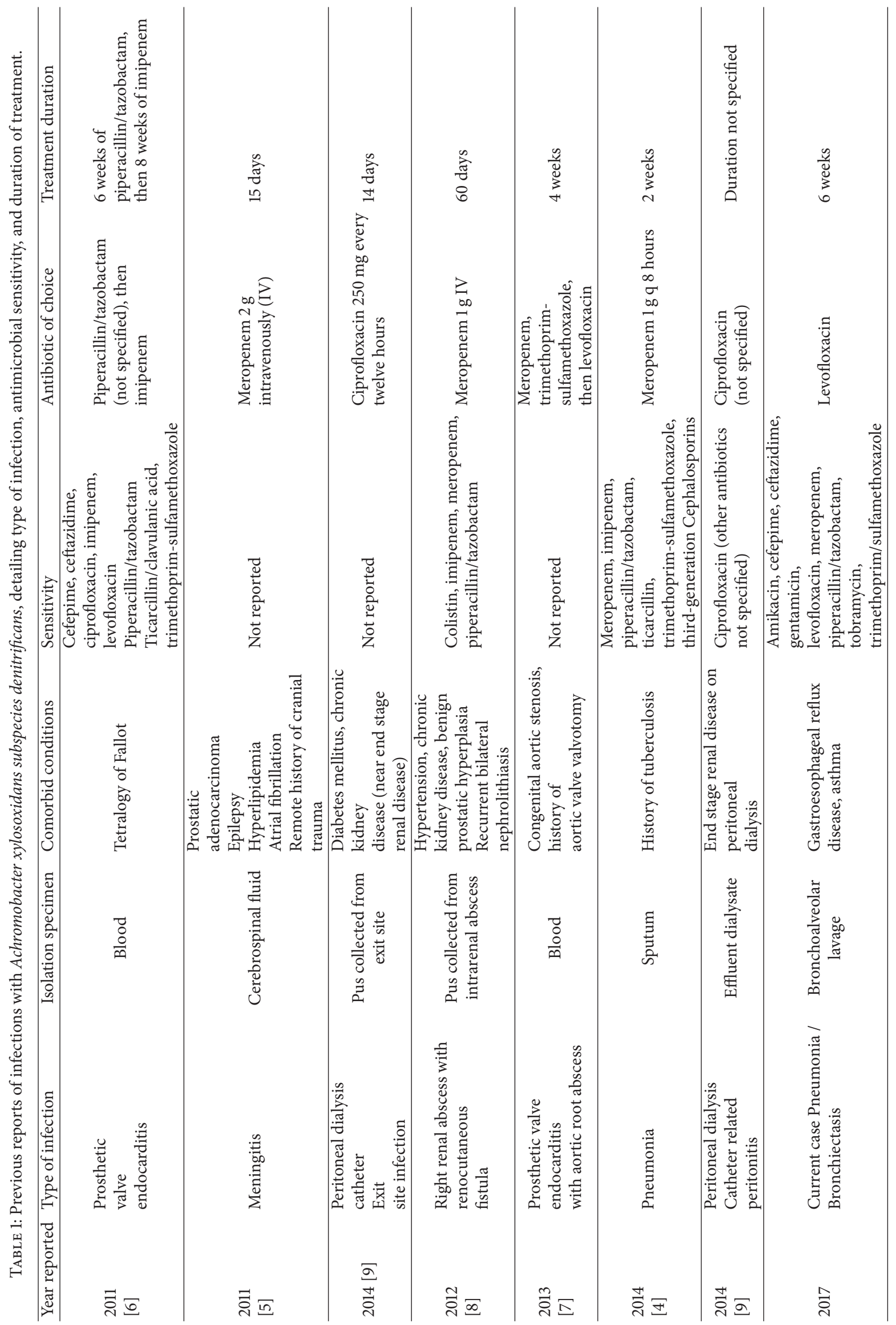




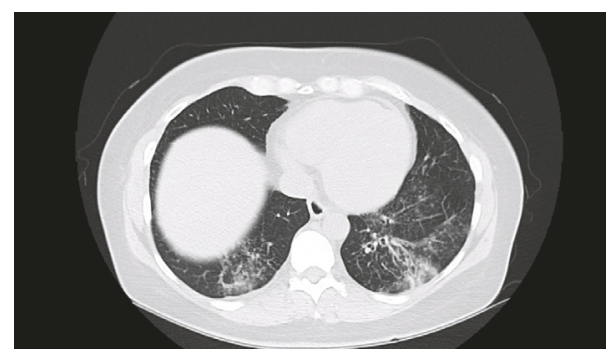

FIGURE 1: Infiltrates and bronchiectatic changes in lower lobes bilaterally.

techniques. She was started again on levofloxacin for two more weeks with good clinical response and no recurrence of symptoms after a total 5 weeks of antibiotic therapy. She was referred for surgical intervention to abolish the ongoing injury to the airway and stop the aspiration insult to the airways.

\section{Discussion}

Achromobacter denitrificans is an aerobic, nonglucose fermenter gram-negative bacillus and flagellated and motile and produces acid from xylose [1]. The genus Achromobacter has multiple subspecies: xylosoxidans, ruhlandii, piechaudii, denitrificans, spanius, insolitus, and marplatensis. The most clinically significant subspecies are Achromobacter xylosoxidans and denitrificans [2]. It can be found in nature in soil, and the xylosoxidans subspecies has an affinity for aquatic surfaces. There are more clinically significant isolates of the subspecies xylosoxidans compared to denitrificans in terms of incidence of infection and clinical variety. Reports about infections with Achromobacter denitrificans are rare as an emerging pathogen.

There is a multitude of respiratory system infection cases due to Achromobacter xylosoxidans, subspecies xylosoxidans but not denitrificans [3]. The first reported pneumonia case due to Achromobacter denitrificans was reported from India in a 48-year-old male clerk in a chemical factory. It was isolated from sputum at two different occasions with no other concomitant isolates. It was sensitive to meropenem, imipenem, piperacillin-tazobactam, ticarcillin, trimethoprim-sulfamethoxazole, and third-generation cephalosporins. It was successfully treated with two weeks of meropenem [4]. Our case is to date the second reported case of Achromobacter xylosoxidans subspecies denitrificans. Other reported infections in adults include meningitis [5], endocarditis [6], endocarditis with aortic root abscess [7], renal abscess [8], peritoneal dialysis catheter related peritonitis [9], and exit site infection [10] (Table 1).

Diagnosis depends on isolation of the organism depending on site of the infection. So far, we have reported isolates from septum, pus, peritoneal fluid, and cerebrospinal fluid (Table 1). Identification can be via standard culturing methods. In our case bronchoalveolar lavage samples have been incubated in Blood Agar (TSA with 5\% Sheep
Blood)/MacConkey Agar Plate using a BioMérieux VITEK-2 system, incubated at 36-37 degrees Celsius.

The immunosuppressed population are at higher risk of infection due to Achromobacter species [3], yet as seen in Table 1 most of the patients had not been overtly immunocompromised but may have had predisposing conditions (end stage renal disease, presence of catheters, etc.). Both cases with involvement of respiratory system had a background of bronchiectasis. The former [4] was most likely secondary to tuberculosis while in our case it is most likely secondary to recurrent aspiration. We theorize that the damaged bronchiectatic lung tissue predisposed to the infection with this organism. This had been illustrated before in that Achromobacter species is known to colonize and infect cystic fibrosis patients [11]. In the other reported cases (Table 1), we can notice that structural damage and/or foreign body had been present: previous trauma, prosthetic valve, peritoneal dialysis catheter, and renal stones. Pathogenicity of Achromobacter species has been previously studied [12] with demonstrated ability to form biofilms and motility (via pili and flagella) which can potentiate infections in setting of structural damage and devices.

Management depends on administration of appropriate antibacterial agents, yet duration of treatment is not exactly defined due to lack in specific guidelines in this regard. The cases reported so far (Table 1) had been managed with different regimens but the most palpable response was to carbapenems with durations ranging from 2 to 14 weeks (Table 1). The sensitivity of the isolates is outlined below in Table 1, but the Achromobacter species had been historically responsive to antipseudomonal agents [1] with various success rates depending on site of infection and complexity of the cases. Our patient was cured after 5 weeks of oral levofloxacin therapy (after initial failure after three weeks). The outcome is generally excellent with clearance of the infection.

\section{Conclusion}

Rare causes of pneumonia should be investigated since appropriate detection can facilitate accurate antibacterial management. We theorize that structural damage (bronchiectasis secondary to chronic aspiration) plays a role in the pathogenesis of pneumonia in our patient. Combined management with antibiotics and airway clearance techniques resulted in an excellent outcome.

\section{Conflicts of Interest}

The authors have no conflicts of interest or financial disclosures to announce.

\section{References}

[1] Y. Glupczynski, W. Hansen, J. Freney, and E. Yourassowsky, "In vitro susceptibility of Alcaligenes denitrificans subsp. xylosoxidans to 24 antimicrobial agents," Antimicrobial Agents and Chemotherapy, vol. 32, no. 2, pp. 276-278, 1988.

[2] T. Coenye, M. Vancanneyt, E. Falsen, J. Swings, and P. Vandamme, "Achromobacter insolitus sp. nov. and Achromobacter 
spanius sp. nov., from human clinical samples," International Journal of Systematic and Evolutionary Microbiology, vol. 53, no. 6, pp. 1819-1824, 2003.

[3] J. M. Duggan, S. J. Goldstein, C. E. Chenoweth, C. A. Kauffman, and S. F. Bradley, "Achromobacter xylosoxidans bacteremia: report of four cases and review of the literature," Clinical Infectious Diseases, vol. 23, no. 3, pp. 569-576, 1996.

[4] A. Bharadiya, M. Mane, S. Pawar, and S. Aundhakar, "“Watch out! Pneumonia secondary to Achromobacter denitrificans", Annals of Medical and Health Sciences Research, vol. 4, no. 7, p. 22, 2014.

[5] P. Manckoundia, E. Mazen, A. S. Coste et al., "A case of meningitis due to achromobacter xylosoxidans denitrificans 60 years after a cranial trauma," Medical Science Monitor, vol. 17, no. 6, pp. CS63-CS65, 2011.

[6] C. Derber, K. Elam, B. A. Forbes, and G. Bearman, "Achromobacter species endocarditis: A case report and literature review," Canadian Journal of Infectious Diseases and Medical Microbiology, vol. 22, no. 3, pp. e17-e20, 2011.

[7] K. Padmaja, V. Lakshmi, M. Amaresh, R. Mishra, R. Chikkala, and V. Sritharan, "Prosthetic Valve Endocarditis with aortic root abscess," International Journal of Infection Control, vol. 9, no. 1, 2013.

[8] A. Sgrelli, A. Mencacci, M. Fiorio, C. Orlandi, F. Baldelli, and G. V. L. De Socio, "Achromobacter denitrificans renal abscess," New Microbiologica, vol. 35, no. 2, pp. 245-247, 2012.

[9] E. Cankaya, M. Keles, E. Gulcan, A. Uyanik, and H. Uyanik, "A rare cause of peritoneal dialysis-related peritonitis: Achromobacter denitrificans," Peritoneal Dialysis International, vol. 34, no. 1, pp. 135-137, 2014.

[10] M. T. Tsai, W. C. Yang, and C. C. Lin, "Continuous ambulatory peritoneal dialysis-related exit-site Infections caused by Achromobacter denitrificans and A. xylosoxidans," Peritoneal Dialysis International, vol. 32, no. 3, pp. 362-363, 2012.

[11] F. De Baets, P. Schelstraete, S. Van Daele, F. Haerynck, and M. Vaneechoutte, "Achromobacter xylosoxidans in cystic fibrosis: Prevalence and clinical relevance," Journal of Cystic Fibrosis, vol. 6, no. 1, pp. 75-78, 2007.

[12] M. Trancassini, V. Iebba, N. Citerà et al., "Outbreak of achromobacter xylosoxidans in an italian cystic fibrosis center: Genome variability, biofilm production, antibiotic resistance, and motility in isolated strains," Frontiers in Microbiology, vol. 5, article no. 138, 2014. 


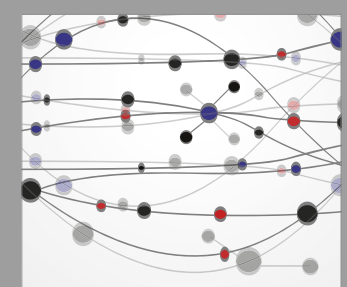

The Scientific World Journal
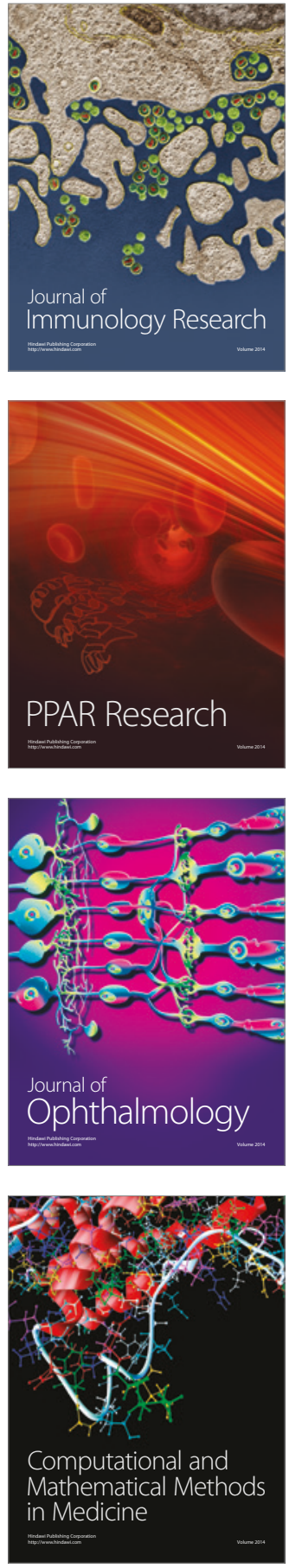

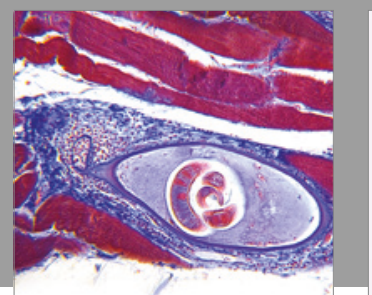

Gastroenterology Research and Practice
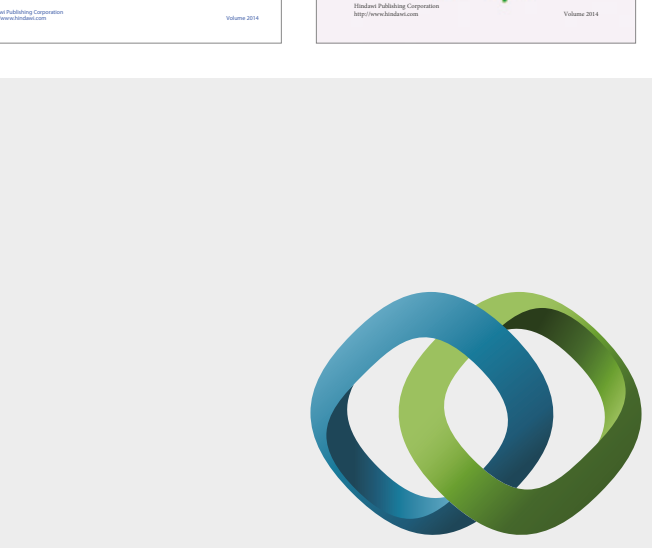

\section{Hindawi}

Submit your manuscripts at

https://www.hindawi.com
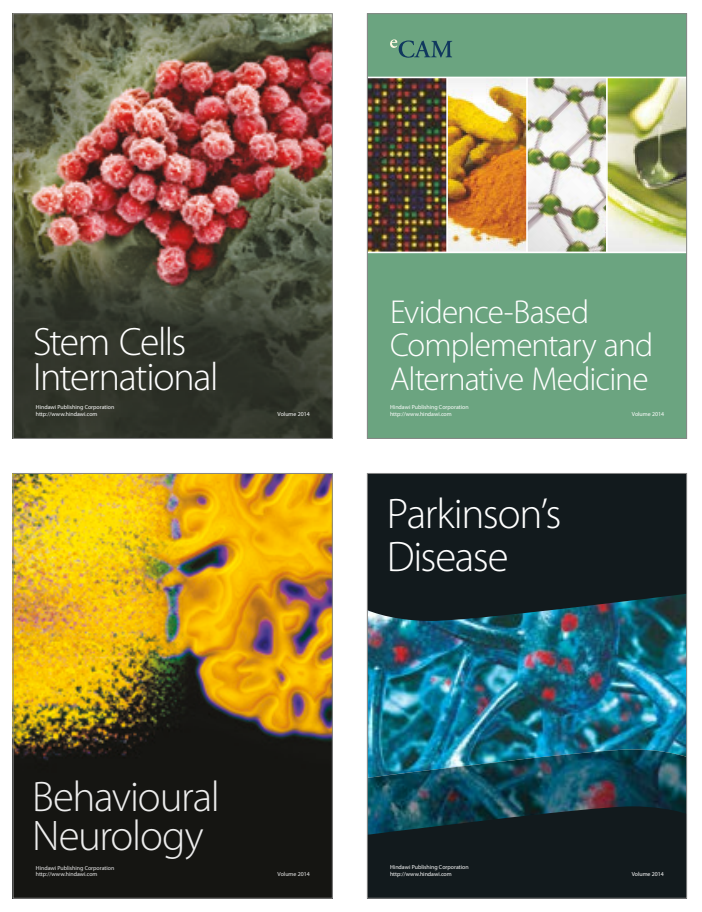
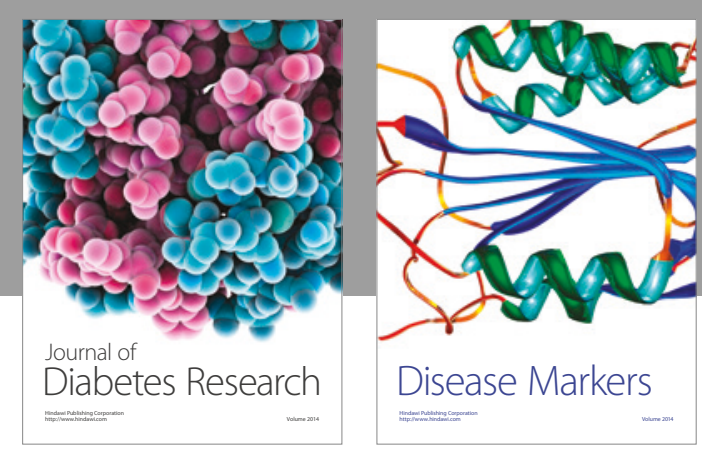

Disease Markers
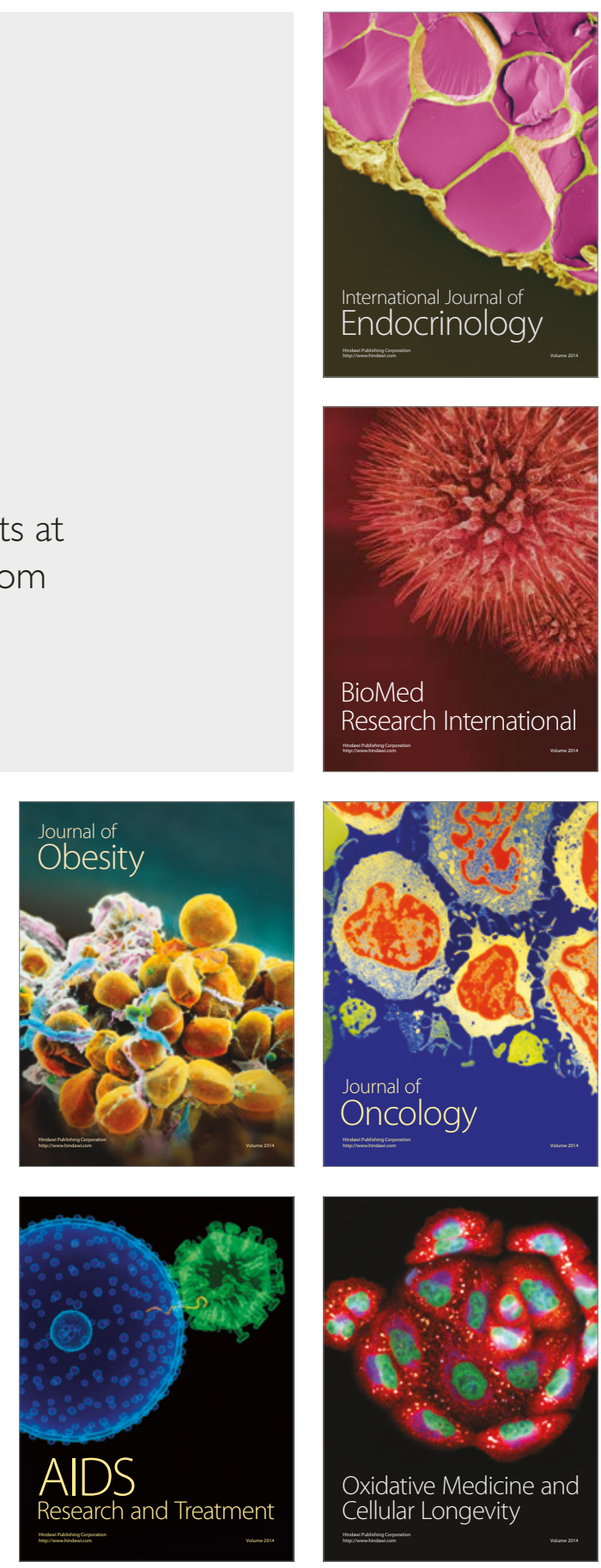